\title{
Approximate controllability of impulsive neutral stochastic differential equations with fractional Brownian motion in a Hilbert space
}

Hamdy M Ahmed*

\section{"Correspondence:}

hamdy_17eg@yahoo.com Higher Institute of Engineering,

El-Shorouk Academy, P.O. 3

El-Shorouk City, Cairo, Egypt

\begin{abstract}
Approximate controllability for impulsive neutral stochastic functional differential equations with finite delay and fractional Brownian motion in a Hilbert space are studied. The results are obtained by using semigroup theory, stochastic analysis, and Banach's fixed point theorem. Finally, an example is given to illustrate the application of our result.
\end{abstract}

MSC: $60 \mathrm{H} 15 ; 60 \mathrm{G} 22 ; 93 \mathrm{~B} 05 ; 34 \mathrm{~A} 37$

Keywords: fractional Brownian motion; approximate controllability; mild solution; impulsive neutral stochastic functional differential equations; fixed point theorem

\section{Introduction}

The impulsive differential systems are used to describe processes which are subjected to abrupt changes at certain moments. The impulsive effects exist widely in the different areas of the real world such as mechanics, electronics, telecommunications, neural networks, finance and economics, etc. (see [1-7]). On the other hand, it is well known that the stochastic control theory is a stochastic generalization of classical control theory. As one of the fundamental concepts in mathematical control theory, controllability plays an important role both in deterministic and stochastic control theory. Controllability generally means that it is possible to steer a dynamical control system from an arbitrary initial state to an arbitrary final state using the set of admissible controls (see [8-17]). Moreover, the approximate controllability means that the system can be steered to arbitrary small neighborhood of final state. Approximate controllable systems are more prevalent and very often approximate controllability is completely adequate in applications (see [1824]).

The purpose of this paper is to investigate the approximate controllability problem for the class of impulsive neutral stochastic functional differential equations with finite delay and fractional Brownian motion in a Hilbert space of the form

$$
\left\{\begin{array}{l}
d[x(t)+g(t, x(t-r(t)))]=[A x(t)+f(t, x(t-v(t)))+B u(t)] d t+\sigma(t) d B^{H}(t), \\
\quad 0 \leq t \leq T, t \neq t_{k}, \\
\left.\Delta x\right|_{t=t_{k}}=I_{k}\left(x\left(t_{k}^{-}\right)\right), \quad k=1,2, \ldots, m \\
x(t)=\varphi(t), \quad-\tau \leq t \leq 0,
\end{array}\right.
$$

O2014 Ahmed; licensee Springer. This is an Open Access article distributed under the terms of the Creative Commons Attribution License (http://creativecommons.org/licenses/by/2.0), which permits unrestricted use, distribution, and reproduction in any medium, provided the original work is properly cited. 
where $A$ is the infinitesimal generator of an analytic semigroup of bounded linear operators, $S(t)_{t \geq 0}$, in a Hilbert space $X, B^{H}$ is a fractional Brownian motion on a real and separable Hilbert space $Y$, the initial data $\varphi \in C\left([-\tau, 0], L^{2}(\Omega, X)\right)$ and the control function $u(\cdot)$ is given in $L^{2}([0, T], U)$, the Hilbert space of admissible control functions with $U$ a Hilbert space. The symbol $B$ stands for a bounded linear from $U$ into $X$. The functions $r, v:[0,+\infty) \rightarrow[0, \tau](\tau>0)$ are continuous, $\left.\Delta x\right|_{t=t_{k}}=I_{k}\left(x\left(t_{k}^{-}\right)\right)$, where $x\left(t_{k}^{+}\right)$and $x\left(t_{k}^{-}\right)$represent the right and left limits of $x(t)$ at $t=t_{k}$, respectively, and $f, g$ : $[0,+\infty) \times X \rightarrow X, \sigma:[0,+\infty) \rightarrow L_{2}^{0}(Y, X)$, are appropriate Lipschitz type functions. Here $R_{T}:=C\left([-\tau, T], L^{2}(\Omega, X)\right)$ be the Banach space of all continuous functions $\xi$ from $[-\tau, T]$ into $L^{2}(\Omega, X)$, equipped with the supremum norm $\|\xi\|_{R_{T}}=\sup _{y \in[-\tau, T]}\left(E\|\xi(y)\|^{2}\right)^{1 / 2}$.

\section{Fractional Brownian motion}

Fix a time interval $[0, T]$ and let $(\Omega, F, P)$ be a complete probability space.

Suppose that $\left\{\beta^{H}(t), t \in[0, T]\right\}$ is the one-dimensional fractional Brownian motion with Hurst parameter $H \in(1 / 2,1)$. That is, $\beta^{H}$ is a centered Gaussian process with covariance function $R_{H}(s, t)=\frac{1}{2}\left(t^{2 H}+s^{2 H}-|t-s|^{2 H}\right)$ (see [25]).

Moreover, $\beta^{H}$ has the following Wiener integral representation:

$$
\beta^{H}(t)=\int_{0}^{t} K_{H}(t, s) d \beta(s)
$$

where $\beta=\{\beta(t), t \in[0, T]\}$ is a Wiener process, and $K_{H}(t, s)$ is the kernel given by

$$
K_{H}(t, s)=c_{H} s^{\frac{1}{2}-H} \int_{s}^{t}(u-s)^{H-\frac{3}{2}} u^{H-\frac{1}{2}} d u
$$

for $s<t$, where $c_{H}=\sqrt{\frac{H(2 H-1)}{\beta\left(2-2 H, H-\frac{1}{2}\right)}}$ and

$$
\beta(p, q)=\int_{0}^{1} t^{p-1}(1-t)^{q-1}, \quad p>0, q>0 .
$$

We put $K_{H}(t, s)=0$ if $t \leq s$.

We will denote by $\zeta$ the reproducing kernel Hilbert space of the $\mathrm{ABm}$. In fact $\zeta$ is the closure of set of indicator functions $\left\{1_{[0, t]}, t \in[0, T]\right\}$ with respect to the scalar product $\left\langle 1_{[0, t]}, 1_{[0, s]}\right\rangle_{\zeta}=R_{H}(t, s)$.

The mapping $1_{[0, t]} \rightarrow \beta^{H}(t)$ can be extended to an isometry from $\zeta$ onto the first Wiener chaos and we will denote by $\beta^{H}(\varphi)$ the image of $\varphi$ under this isometry.

We recall that for $\psi, \varphi \in \zeta$ their scalar product in $\zeta$ is given by

$$
\langle\psi, \varphi\rangle_{\zeta}=H(2 H-1) \int_{0}^{T} \int_{0}^{T} \psi(s) \varphi(t)|t-s|^{2 H-2} d s d t
$$

Let us consider the operator $K^{*}$ from $\zeta$ to $L^{2}([0, T])$ defined by

$$
\left(K_{H}^{*} \varphi\right)(s)=\int_{S}^{T} \varphi(r) \frac{\partial K}{\partial r}(r, s) d r
$$


Moreover, for any $\varphi \in \zeta$, we have

$$
\beta^{H}(\varphi)=\int_{0}^{T}\left(K_{H}^{*} \varphi\right)(t) d \beta(t) .
$$

Let $X$ and $Y$ be two real, separable Hilbert spaces and let $L(Y, X)$ be the space of bounded linear operators from $Y$ to $X$. For the sake of convenience, we shall use the same notation to denote the norms in $X, Y$ and $L(Y, X)$. Let $Q \in L(Y, Y)$ be an operator defined by $Q e_{n}=$ $\lambda_{n} e_{n}$ with finite trace $\operatorname{tr} Q=\sum_{n=1}^{\infty} \lambda_{n}<\infty$, where $\lambda_{n} \geq 0(n=1,2, \ldots)$ are non-negative real numbers and $\left\{e_{n}\right\}(n=1,2, \ldots)$ is a complete orthonormal basis in $Y$.

We define the infinite-dimensional $\mathrm{fBm}$ on $Y$ with covariance $Q$ as

$$
B^{H}(t)=B_{Q}^{H}(t)=\sum_{n=1}^{\infty} \sqrt{\lambda_{n}} e_{n} \beta_{n}^{H}(t),
$$

where $\beta_{n}^{H}$ are real, independent fBm's. The $Y$-valued process is Gaussian, starts from 0 , has mean zero and covariance:

$$
E\left\langle B^{H}(t), x\right\rangle\left\langle B^{H}(s), y\right\rangle=R(s, t)\langle Q(x), y\rangle \quad \text { for all } x, y \in Y \text { and } t, s \in[0, T]
$$

In order to define Wiener integrals with respect to the $Q-\mathrm{fBm}$, we introduce the space $L_{2}^{0}:=L_{2}^{0}(Y, X)$ of all $Q$-Hilbert Schmidt operators $\psi: Y \rightarrow X$. We recall that $\psi \in L(Y, X)$ is called a $Q$-Hilbert-Schmidt operator, if

$$
\|\psi\|_{L_{2}^{0}}^{2}:=\sum_{n=1}^{\infty}\left\|\sqrt{\lambda}_{n} \psi e_{n}\right\|^{2}<\infty
$$

and that the space $L_{2}^{0}$ equipped with the inner product $\langle\varphi, \psi\rangle_{L_{2}^{0}}=\sum_{n=1}^{\infty}\left\langle\varphi e_{n}, \psi e_{n}\right\rangle$ is a separable Hilbert space.

Let $\phi(s) ; s \in[0, T]$ be a function with values in $L_{2}^{0}(Y, X)$, the Wiener integral of $\phi$ with respect to $B^{H}$ is defined by

$$
\begin{aligned}
\int_{0}^{t} \phi(s) d B^{H}(s) & =\sum_{n=1}^{\infty} \int_{0}^{t} \sqrt{\lambda} \phi(s) e_{n} d \beta_{n}^{H} \\
& =\sum_{n=1}^{\infty} \int_{0}^{t} \sqrt{\lambda} K^{*}\left(\phi e_{n}\right)(s) d \beta_{n}(s),
\end{aligned}
$$

where $\beta_{n}$ is the standard Brownian motion.

Lemma 2.1 (see [26]) If $\psi:[0, T] \rightarrow L_{2}^{0}(Y, X)$ satisfies $\int_{0}^{T}\|\psi(s)\|_{L_{2}^{0}}^{2}<\infty$ then the above sum in (2.1) is well defined as $X$-valued random variable and we have

$$
E\left\|\int_{0}^{t} \psi(s) d B^{H}(s)\right\|^{2} \leq 2 H t^{2 H-1} \int_{0}^{t}\|\psi(s)\|_{L_{2}^{0}}^{2} d s .
$$




\section{Approximate controllability}

Let $A: D(A) \rightarrow X$ be the infinitesimal generator of an analytic semigroup, $(S(t))_{t \geq 0}$, of bounded linear operators on $X$. It is well known that there exist $M \geq 1$ and $\lambda \in R$ such that $\|S(t)\| \leq M e^{\lambda t}$ for every $t \geq 0$.

If $(S(t))_{t \geq 0}$ is uniformly bounded and analytic semigroup such that $0 \in \rho(A)$, where $\rho(A)$ is the resolvent set of $A$, then it is possible to define the fractional power $(-A)^{\alpha}$ for $0<\alpha \leq 1$, as a closed linear operator on its domain $D(-A)^{\alpha}$. Furthermore, the subspace $D(-A)^{\alpha}$ is dense in $X$ and the expression $\|h\|_{\alpha}=\left\|(-A)^{\alpha} h\right\|$ defines a norm in $D(-A)^{\alpha}$. If $X_{\alpha}$ represents the space $D(-A)^{\alpha}$ endowed with the norm $\|\cdot\|_{\alpha}$, then the following properties are well known.

Lemma 3.1 ([27])

(1) Let $0<\alpha \leq 1$, then $X_{\alpha}$ is a Banach space.

(2) If $0<\beta \leq \alpha$, then the injection $X_{\alpha} \hookrightarrow X_{\beta}$ is continuous.

(3) For every $0<\alpha \leq 1$ there exists $M_{\alpha}>0$ such that

$$
\left\|(-A)^{\alpha} S(t)\right\| \leq M_{\alpha} t^{-\alpha} e^{-\lambda t}, \quad t>0, \lambda>0 .
$$

Now, we present the mild solution of the problem (1.1):

Definition 3.1 An $X$-valued process $\{x(t), t \in[-\tau, T]\}$ is called a mild solution of equation (1.1) if

(i) $x(\cdot) \in C\left([-\tau, T], L^{2}(\Omega, X)\right)$,

(ii) $x(t)=\varphi(t),-\tau \leq t \leq 0$,

(iii) for arbitrary $t \in[0, T]$, we have

$$
\begin{aligned}
x(t)= & S(t)[\varphi(0)+g(0, \varphi(-r(0)))]-g(t, x(t-r(t))) \\
& -\int_{0}^{t} A S(t-s) g(s, x(s-r(s))) d s \\
& +\int_{0}^{t} S(t-s) f(s, x(s-v(s))) d s+\int_{0}^{t} S(t-s) B u(s) d s \\
& +\int_{0}^{t} S(t-s) \sigma(s) d B^{H}(s)+\sum_{0<t_{k}<t} S\left(t-t_{k}\right) I_{k}\left(x\left(t_{k}^{-}\right)\right) .
\end{aligned}
$$

In this paper, we will make the following assumptions.

(H1) The operator $A$ is the infinitesimal generator of an analytic semigroup, $(S(t))_{t \geq 0}$, consisting of bounded linear operators on $X$. Furthermore, there exist constants $M$ and $M_{1-\beta}$ such that for every $t \in[0, T]$ the inequalities $\|S(t)\| \leq M$ and $t^{1-\beta}\left\|(-A)^{1-\beta} S(t)\right\| \leq$ $M_{1-\beta}$ hold.

(H2) There exist finite positive constants $C_{i}=C_{i}(T), i=1,2$, such that the function $f$ : $[0,+\infty) \times X \rightarrow X$ satisfies the following Lipschitz conditions: for all $t \in[0, T]$ and $x, y \in X$ the inequalities $\|f(t, x)-f(t, y)\| \leq C_{1}\|x-y\|$ and $\|f(t, x)\|^{2} \leq C_{2}^{2}\left(1+\|x\|^{2}\right)$ are valid.

(H3) The function $g$ is $X_{\beta}$-valued, and there exist constants $\frac{1}{2}<\beta<1, C_{i}=C_{i}(T), i=3,4$, such that for all $t \in[0, T]$ and $x, y \in X$ the following inequalities are satisfied:

(i) $\left\|(-A)^{\beta} g(t, x)-(-A)^{\beta} g(t, y)\right\| \leq C_{3}\|x-y\|$;

(ii) $\left\|(-A)^{\beta} g(t, x)\right\|^{2} \leq C_{4}^{2}\left(1+\|x\|^{2}\right)$;

(iii) $C_{3}\left\|(-A)^{-\beta}\right\|<1$. 
(H4) The function $(-A)^{\beta} g$ is continuous in the quadratic mean sense: for all $x \in$ $C\left([0, T], L^{2}(\Omega, X)\right)$, the equality

$$
\lim _{t \rightarrow s} E\left\|(-A)^{\beta} g(t, x(t))-(-A)^{\beta} g(s, x(s))\right\|^{2}=0
$$

is true.

(H5) The function $\sigma:[0, \infty) \rightarrow L_{2}^{0}(Y, X)$ satisfies $\int_{0}^{T}\|\sigma(s)\|_{L_{2}^{0}}^{2} d s<\infty$.

(H6) The functions $I_{k}: X \rightarrow X$ are continuous and there exist finite positive constants $C_{i}=C_{i}(T), i=5,6$, such that for all $t \in[0, T]$ and $x, y \in X$ the inequalities $\| I_{k}(x(t))-$ $I_{k}(y(t))\left\|\leq C_{5}\right\| x-y \|$ and $\left\|I_{k}(x(t))\right\|^{2} \leq C_{6}^{2}\left(1+\|x\|^{2}\right)$ are valid.

In order to study the approximate controllability for the system (1.1), we introduce the following linear differential system:

$$
\left\{\begin{array}{l}
\frac{d x(t)}{d t}=A x(t)+B u(t), \quad t \in[0, T], \\
x(0)=x_{0} .
\end{array}\right.
$$

The controllability operator associated with (3.2) is defined by

$$
\Gamma_{0}^{T}=\int_{0}^{T} S(T-s) B B^{*} S^{*}(T-s) d s
$$

where $B^{*}$ and $S^{*}$ denote the adjoint of $B$ and $S$, respectively.

Let $x(T ; \varphi, u)$ be the state value of $(1.1)$ at terminal state $T$, corresponding to the control $u$ and the initial value $\varphi$. Denote by $R(T, \varphi)=\left\{x(T ; \varphi, u): u \in L^{2}([0, T], U)\right\}$ the reachable set of system (1.1) at terminal time $T$, its closure in $X$ is denoted by $\overline{R(T, \varphi)}$.

Definition 3.2 The system (1.1) is said to be approximately controllable on the interval $[0, T]$ if $\overline{R(T, \varphi)}=L^{2}(\Omega, X)$.

Lemma 3.2 (see [19]) The linear control system (3.2) is approximately controllable on $[0, T]$ if and only if $z\left(z I+\Gamma_{0}^{T}\right)^{-1} \rightarrow 0$ strongly as $z \rightarrow 0^{+}$.

Lemma 3.3 For any $\bar{x}_{T} \in L^{2}(\Omega, X)$ there exists $\bar{\varphi} \in L^{2}\left(\Omega ; L^{2}\left([0, T] ; L_{2}^{0}\right)\right)$ such that

$$
\bar{x}_{T}=E \bar{x}_{T}+\int_{0}^{T} \bar{\varphi}(s) d B^{H}(s) .
$$

Now for any $\delta>0$ and $\bar{x}_{T} \in L^{2}(\Omega, X)$, we define the control function in the following form:

$$
\begin{aligned}
u^{\delta}(t, x)= & B^{*} S^{*}(T-t)\left(z I+\Gamma_{0}^{T}\right)^{-1} \\
& \times\left\{E \bar{x}_{T}-S(T)[\varphi(0)-g(0, \varphi(-r(0)))]+g(T, x(T))\right\} \\
& +B^{*} S^{*}(T-t) \int_{0}^{t}\left(z I+\Gamma_{0}^{T}\right)^{-1} \bar{\varphi}(s) d B^{H}(s) \\
& -B^{*} S^{*}(T-t) \int_{0}^{t}\left(z I+\Gamma_{0}^{T}\right)^{-1} A S(T-s) g(s, x(s-r(s))) d s \\
& -B^{*} S^{*}(T-t) \int_{0}^{t}\left(z I+\Gamma_{0}^{T}\right)^{-1} S(T-s) f(s, x(s-v(s))) d s
\end{aligned}
$$




$$
\begin{aligned}
& -B^{*} S^{*}(T-t) \int_{0}^{t}\left(z I+\Gamma_{0}^{T}\right)^{-1} S(T-s) \sigma(s) d B^{H}(s) \\
& -B^{*} S^{*}(T-t) \sum_{0<t_{k}<t}\left(z I+\Gamma_{0}^{T}\right)^{-1} S\left(T-t_{k}\right) I_{k}\left(x\left(t_{k}^{-}\right)\right) .
\end{aligned}
$$

Lemma 3.4 There exists a positive real constant $M_{C}$ such that, for all $x, y \in R_{T}$, we have

$$
\begin{aligned}
& E\left\|u^{\delta}(t, x)-u^{\delta}(t, y)\right\|^{2} \leq \frac{M_{C}}{z^{2}} \int_{0}^{t} E\|x(s)-y(s)\|^{2} d s, \\
& E\left\|u^{\delta}(t, x)\right\|^{2} \leq \frac{M_{C}}{z^{2}}\left(1+\int_{0}^{t} E\|x(s)\|^{2} d s\right) .
\end{aligned}
$$

Proof The proof of this lemma similar to the proof of the Lemma 2.5 (see [28]).

Theorem 3.1 Assume assumptions (H1)-(H6) are satisfied. Then, for all $T>0$, the system (1.1) has a mild solution on $[-\tau, T]$.

Proof Fix $T>0$ and let us consider $\Upsilon_{T}=\left\{x \in R_{T}: x(s)=\varphi(s)\right.$, for $\left.s \in[-\tau, 0]\right\}$.

$\Upsilon_{T}$ is a closed subset of $R_{T}$ provided with the norm $\|\cdot\|_{R_{T}}$. For any $\delta>0$, consider the operator $\Pi_{\delta}$ on $R_{T}$ defined as follows:

$$
\left(\Pi_{\delta} x\right)(t)=\left\{\begin{array}{l}
\varphi(t), \quad t \in[-\tau, 0], \\
S(t)[\varphi(0)+g(0, \varphi(-r(0)))]-g(t, x(t-r(t))) \\
\quad-\int_{0}^{t} A S(t-s) g(s, x(s-r(s))) d s \\
\quad+\int_{0}^{t} S(t-s) f(s, x(s-v(s))) d s+\int_{0}^{t} S(t-s) B u^{\delta}(s, x) d s \\
\quad+\int_{0}^{t} S(t-s) \sigma(s) d B^{H}(s)+\sum_{0<t_{k}<t} S\left(t-t_{k}\right) I_{k}\left(x\left(t_{k}^{-}\right)\right), \quad t \in[0, T] .
\end{array}\right.
$$

It will be shown that, for all $\delta>0$, the operator $\Pi_{\delta}$ has a fixed point. This fixed point is then a solution of equation (1.1). To prove this result, we divide the subsequent proof into two steps.

Step 1: For arbitrary $x \in \Upsilon_{T}$, let us prove that $t \rightarrow \Pi_{\delta}(x)(t)$ is continuous on the interval $[0, T]$ in the $L^{2}(\Omega, X)$-sense.

Let $0<t<t+h<T$, where $t, t+h \in[0, T] \backslash\left\{t_{1}, t_{2}, \ldots, t_{m}\right\}$, and let $|h|$ be sufficiently small. Then for any fixed $x \in \Upsilon_{T}$, it follows from Holder's inequality and the assumptions on the theorem that

$$
\begin{aligned}
& E\left\|\left(\Pi_{\delta} x\right)(t+h)-\left(\Pi_{\delta} x\right)(t)\right\|^{2} \\
& \leq 8\left\{E\|(S(t+h)-S(t))(\varphi(0)+g(0, \varphi(-r(0))))\|^{2}\right. \\
& +E\|g(t+h, x(t+h-r(t)))-g(t, x(t-r(t)))\|^{2} \\
& +E\left\|\int_{0}^{t} A(S(t+h-s)-S(t-s)) g(s, x(s-r(s))) d s\right\|^{2} \\
& +E\left\|\int_{t}^{t+h}(-A)^{1-\beta} S(t+h-s)(-A)^{\beta} g(s, x(s-r(s))) d s\right\|^{2} \\
& +E\left\|\int_{0}^{t}(S(t+h-s)-S(t-s)) B u^{\delta}(s, x) d s\right\|^{2}+E\left\|\int_{t}^{t+h} S(t+h-s) B u^{\delta}(s, x) d s\right\|^{2}
\end{aligned}
$$




$$
\begin{aligned}
& +E\left\|\int_{0}^{t}(S(t+h-s)-S(t-s)) \sigma(s) d B^{H}(s)\right\|^{2}+E\left\|\int_{t}^{t+h} S(t+h-s) \sigma(s) d B^{H}(s)\right\|^{2} \\
& +\sum_{0<t_{k}<t} E\left\|\left(S\left(t+h-t_{k}\right)-S\left(t-t_{k}\right)\right) I_{k}\left(x\left(t_{k}^{-}\right)\right)\right\|^{2} \\
& \left.+\sum_{t<t_{k}<t+h} E\left\|S\left(t+h-t_{k}\right) I_{k}\left(x\left(t_{k}^{-}\right)\right)\right\|^{2}\right\} \\
& \leq 8\left\{E\|(S(t+h)-S(t))(\varphi(0)+g(0, \varphi(-r(0))))\|^{2}\right. \\
& +E\|g(t+h, x(t+h-r(t)))-g(t, x(t-r(t)))\|^{2} \\
& +t \int_{0}^{t} E\|A(S(t+h-s)-S(t-s)) g(s, x(s-r(s)))\|^{2} d s \\
& +\frac{C_{4}^{2} M_{1-\beta}^{2}}{2 \beta-1}(t+h-t)^{2 \beta-1} \int_{t}^{t+h}\left(1+E\|x\|^{2}\right) d s \\
& +t \int_{0}^{t} E\left\|(S(t+h-s)-S(t-s)) B u^{\delta}(s, x)\right\|^{2} d s \\
& +(t+h-t) M^{2}\|B\|^{2} \int_{t}^{t+h} E\left\|u^{\delta}(s, x)\right\|^{2} d s \\
& +2 H t^{2 H-1} \int_{0}^{t} E\|(S(t+h-s)-S(t-s)) \sigma(s)\|_{L_{2}^{0}}^{2} d s \\
& +2 H(t+h-t)^{2 H-1} M^{2} \int_{t}^{t+h} E\|\sigma(s)\|_{L_{2}^{0}}^{2} d s \\
& \left.+\sum_{0<t_{k}<t} E\left(\left\|\left(S\left(t+h-t_{k}\right)-S\left(t-t_{k}\right)\right)\right\|^{2}\left\|I_{k}\left(x\left(t_{k}^{-}\right)\right)\right\|^{2}\right)+M^{2} \sum_{t<t_{k}<t+h} E\left\|I_{k}\left(x\left(t_{k}^{-}\right)\right)\right\|^{2}\right\} .
\end{aligned}
$$

Hence using the strong continuity of $S(t)$ and Lebesgue's dominated convergence theorem, we conclude that the right-hand side of the above inequalities tends to zero as $h \rightarrow 0$. Thus we conclude $\Pi_{\delta}(x)(t)$ is continuous from the right in $[0, T]$. A similar argument shows that it is also continuous from the left in $(0, T]$. Thus $\Pi_{\delta}(x)(t)$ is continuous on $[0, T]$ in the $L^{2}$ sense.

Step 2: Now, we are going to show that $\Pi_{\delta}$ is a contraction mapping in $\Upsilon_{T_{1}}$ with some $T_{1} \leq T$ to be specified latter. Let $x, y \in \Upsilon_{T}$, we obtain for any fixed $t \in[0, T]$

$$
\begin{aligned}
& \left\|\Pi_{\delta}(x)(t)-\Pi_{\delta}(y)(t)\right\|^{2} \\
& \leq 5 \| g\left(t, x(t-r(t))-g\left(t, y(t-r(t))\left\|^{2}+5\right\| \int_{0}^{t} S(t-s) B\left[u^{\delta}(s, x)-u^{\delta}(s, y)\right] d s \|^{2}\right.\right. \\
& \quad+5 \| \int_{0}^{t}(-A)^{1-\beta} S(t-s)\left((-A)^{\beta} g\left(s, x(s-r(s))-(-A)^{\beta} g(s, y(s-r(s)))\right) d s \|^{2}\right. \\
& \quad+5\left\|\int_{0}^{t} S(t-s)(f(s, x(s-v(s)))-f(s, y(s-v(s)))) d s\right\|^{2} \\
& \quad+5\left\|\sum_{0<t_{k}<t} S\left(t-t_{k}\right)\left(I_{k}\left(x\left(t_{k}^{-}\right)\right)-I_{k}\left(y\left(t_{k}^{-}\right)\right)\right)\right\|^{2} .
\end{aligned}
$$


By the Lipschitz property of $(-A)^{\beta} g$ and $f$ combined with Holder's inequality, we obtain

$$
\begin{aligned}
E\left\|\Pi_{\delta}(x)(t)-\Pi_{\delta}(y)(t)\right\|^{2} \leq & 5 E x(t-r(t))-y(t-r(t)) \|^{2} \\
& +\frac{5 M^{2}\|B\|^{2} M_{C}}{z^{2}} \int_{0}^{t} E\|x(s)-y(s)\|^{2} d s \\
& +5 C_{3}^{2} M_{1-\beta}^{2} \frac{T^{2 \beta-1}}{2 \beta-1} \int_{0}^{t} E\|x(s-r(s))-y(s-r(s))\|^{2} d s \\
& +5 T C_{1}^{2} M^{2} \int_{0}^{t} E\|x(s-v(s))-y(s-v(s))\|^{2} d s \\
& +5 m^{2} M^{2} C_{5}^{2} E\|x(t)-y(t)\|^{2} .
\end{aligned}
$$

Hence

$$
\sup _{t \in[-\tau, T]} E\left\|\Pi_{\delta}(x)(t)-\Pi_{\delta}(y)(t)\right\|^{2} \leq \gamma(T) \sup _{t \in[-\tau, T]} E\|x(t)-y(t)\|^{2}
$$

where

$$
\gamma(T)=5\left[1+\frac{M^{2}\|B\|^{2} M_{C}}{z^{2}} T+\frac{C_{3}^{2} M_{1-\beta}^{2}}{2 \beta-1} T^{2 \beta}+M^{2} C_{1}^{2} T^{2}+m^{2} M^{2} C_{5}^{2}\right] .
$$

Then there exists $0<T_{1} \leq T$ such that $0<\gamma\left(T_{1}\right)<1$ and $\Pi_{\delta}$ is a contraction mapping on $S_{T_{1}}$ and therefore has a unique fixed point, which is a mild solution of equation (1.1) on $\left[-\tau, T_{1}\right]$. This procedure can be repeated in order to extend the solution to the entire interval $[-\tau, T]$ in finitely many steps. This completes the proof.

Theorem 3.2 Assume that (H1)-(H6) are satisfied. Further, if the functions $f$ and $g$ are uniformly bounded, and $S(t)$ is compact, then the system (1.1) is approximately controllable on $[0, T]$.

Proof Let $x_{\delta}$ be a fixed point of $\Pi_{\delta}$. By using the stochastic Fubini theorem, it can easily be seen that

$$
\begin{aligned}
x_{\delta}(T)= & \bar{x}_{T}-z\left(z I+\Gamma_{0}^{T}\right)^{-1}\left\{E \bar{x}_{T}-S(T)[\varphi(0)-g(0, \varphi(-r(0)))]\right. \\
& \left.+g\left(T, x_{\delta}(T)\right)+\int_{0}^{T} \bar{\varphi}(s) d B^{H}(s)\right\} \\
& +z \int_{0}^{T}\left(z I+\Gamma_{0}^{T}\right)^{-1} A S(T-s) g\left(s, x_{\delta}(s-r(s))\right) d s \\
& +z \int_{0}^{T}\left(z I+\Gamma_{0}^{T}\right)^{-1} S(T-s) f\left(s, x_{\delta}(s-v(s))\right) d s \\
& +z \int_{0}^{T}\left(z I+\Gamma_{0}^{T}\right)^{-1} S(T-s) \sigma(s) d B^{H}(s) \\
& +\sum_{0<t_{k}<T} z\left(z I+\Gamma_{0}^{T}\right)^{-1} S\left(T-t_{k}\right) I_{k}\left(x_{\delta}\left(t_{k}^{-}\right)\right) .
\end{aligned}
$$


It follows from the assumption on $f$ and $g$ that there exists $\bar{D}>0$ such that

$$
\left\|f\left(s, x_{\delta}(s-v(s))\right)\right\|^{2}+\left\|g\left(s, x_{\delta}(s-r(s))\right)\right\|^{2} \leq \bar{D}
$$

for all $(s, \omega) \in[0, T] \times \Omega$. Then there is a subsequence still denoted by $\left\{f\left(s, x_{\delta}(s-\right.\right.$ $\left.v(s))), g\left(s, x_{\delta}(s-r(s))\right)\right\}$ which converges weakly to, say, $\{f(s), g(s)\}$ in $X \times L_{2}^{0}$.

From the above equation, we have

$$
\begin{aligned}
& E\left\|x_{\delta}(T)-\bar{x}_{T}\right\|^{2} \\
& \leq 7 E \| z\left(z I+\Gamma_{0}^{T}\right)^{-1}\left\{\bar{x}_{T}-S(T)[\varphi(0)-g(0, \varphi(-r(0)))]\right. \\
& \left.\quad+g\left(T, x_{\delta}(T)\right)+\int_{0}^{T} \bar{\varphi}(s) d B^{H}(s)\right\} \|^{2} \\
& \quad+7 E\left(\int_{0}^{T}\left\|z\left(z I+\Gamma_{0}^{T}\right)^{-1}\right\|\left\|A S(T-s)\left[g\left(s, x_{\delta}(s-r(s))\right)-g(s)\right]\right\| d s\right)^{2} \\
& \quad+7 E\left(\int_{0}^{T}\left\|z\left(z I+\Gamma_{0}^{T}\right)^{-1} A S(T-s) g(s)\right\| d s\right)^{2} \\
& \quad+7 E\left(\int_{0}^{T}\left\|z\left(z I+\Gamma_{0}^{T}\right)^{-1}\right\|\left\|S(T-s) f\left(s, x_{\delta}(s-v(s))\right)-f(s)\right\| d s\right)^{2} \\
& \quad+7 E\left(\int_{0}^{T}\left\|z\left(z I+\Gamma_{0}^{T}\right)^{-1} S(T-s) f(s)\right\| d s\right)^{2} \\
& \quad+14 H T^{2 H-1} \int_{0}^{T}\left\|z\left(z I+\Gamma_{0}^{T}\right)^{-1} S(T-s) \sigma(s)\right\|_{L_{2}^{0}}^{2} d s \\
& \quad+7 E\left\|\sum_{0<t_{k}<T} z\left(z I+\Gamma_{0}^{T}\right)^{-1} S\left(T-t_{k}\right) I_{k}\left(x_{\delta}\left(t_{k}^{-}\right)\right)\right\|^{2} .
\end{aligned}
$$

On the other hand, by Lemma 3.2, the operator $z\left(z I+\Gamma_{0}^{T}\right)^{-1} \rightarrow 0$ strongly as $z \rightarrow 0^{+}$for all $0 \leq s \leq T$, and, moreover, $\left\|z\left(z I+\Gamma_{0}^{T}\right)^{-1}\right\| \leq 1$. Thus, by the Lebesgue dominated convergence theorem the compactness of $S(t)$ implies that $E\left\|x_{\delta}(T)-\bar{x}_{T}\right\|^{2} \rightarrow 0$ as $z \rightarrow 0^{+}$. This gives the approximate controllability of (1.1).

\section{Example}

In this section, we present an example to illustrate our main result.

Let us consider the following stochastic control partial neutral functional differential equation with finite variable delays driven by a fractional Brownian motion:

$$
\left\{\begin{array}{l}
d[x(t, \xi)-G(t, x(t-r(t)), \xi)]=\left[\frac{\partial^{2} x(t, \xi)}{\partial \xi^{2}}+F(t, x(t-v(t)), \xi)+\mu(t, \xi)\right] d t \\
\quad+\sigma(t) d B^{H}(t), \quad 0 \leq \xi \leq \pi, 0 \leq t \leq T, t \neq t_{k}, \\
x(t, 0)=x(t, \pi)=0, \quad t \geq 0, \\
x\left(t_{k}^{+}, \xi\right)-x\left(t_{k}^{-}, \xi\right)=I_{k}\left(x\left(t_{k}^{-}, \xi\right)\right), \quad k=1,2, \ldots, m, \\
x(t, \xi)=\varphi(t, \xi), \quad t \in[-\tau, 0], 0 \leq \xi \leq \pi,
\end{array}\right.
$$

where $B^{H}$ is a fractional Brownian motion and $F, G: R^{+} \times R \rightarrow R$ are continuous functions. 
To study this system, let $X=Y=U=L^{2}([0, \pi], R)$ and let the operator $A: D(A) \subset X \rightarrow X$ be given by $A y=y^{\prime \prime}$ with

$$
D(A)=\left\{y \in X: y^{\prime \prime} \in X, y(0)=y(\pi)=0\right\} .
$$

It is well known that $A$ is the infinitesimal generator of an analytic semigroup $\{T(t)\}_{t \geq 0}$ on $X$. Furthermore, $A$ has discrete spectrum with eigenvalues $-n^{2}, n \in N$ and the corresponding normalized eigenfunctions are given by

$$
e_{n}=\sqrt{\frac{2}{\pi}} \sin n x, \quad n=1,2, \ldots
$$

In addition $\left(e_{n}\right)_{n \in N}$ is a complete orthonormal basis in $X$ and

$$
T(t) x=\sum_{n=1}^{\infty} e^{-n^{2} t}\left\langle x, e_{n}\right\rangle_{e_{n}}
$$

for $x \in X$ and $t \geq 0$. It follows from this representation that $T(t)$ is compact for every $t>0$ and that $\|T(t)\| \leq e^{-t}$ for every $t \geq 0$.

In order to define the operator $Q: Y \rightarrow R$, we choose a sequence $\left\{\lambda_{n}\right\}_{n \in N} \subset R^{+}$, set $Q e_{n}=$ $\lambda_{n} e_{n}$, and assume that

$$
\operatorname{tr}(Q)=\sum_{n=1}^{\infty} \sqrt{\lambda_{n}}<\infty
$$

Define the fractional Brownian motion in $Y$ by

$$
B^{H}(t)=\sum_{n=1}^{\infty} \sqrt{\lambda_{n}} \beta^{H}(t) e_{n}
$$

where $H \in\left(\frac{1}{2}, 1\right)$ and $\left\{\beta_{n}^{H}\right\}_{n \in N}$ is a sequence of one-dimensional fractional Brownian motions mutually independent.

Define $x(t)(\cdot)=x(t, \cdot), f(t, x)(\cdot)=F(t, x(\cdot))$, and $g(t, x)(\cdot)=G(t, x(\cdot))$. Define the bounded operator $B: U \rightarrow X$ by $B u(t)(\xi)=\mu(t, \xi), 0 \leq \xi \leq \pi, u \in U$. Therefore, with the above choice, the system (4.1) can be written into the abstract form (1.1) and all conditions of Theorem 3.2 are satisfied. Thus by Theorem 3.2, the stochastic partial neutral functional differential equation with finite variable delays driven by a fractional Brownian motion is approximately controllable on $[0, \pi]$. 


\section{References}

1. Bainov, D, Simeonov, P: Impulsive Differential Equations: Periodic Solutions and Applications. Longman, Harlow (1993)

2. Rogovchenko, YV: Nonlinear impulsive evolution systems and applications to population models. J. Math. Anal. Appl. 207, 300-315 (1997)

3. Benchohra, M, Henderson, J, Ntouyas, SK: Existence results for impulsive multivalued semilinear neutral functional inclusions in Banach spaces. J. Math. Anal. Appl. 263, 763-780 (2001)

4. Benchohra, M, Henderson, J, Ntouyas, SK: Impulsive Differential Equations and Inclusions. Hindawi Publishing Corporation, New York (2006)

5. Bogdan, P, Marculescu, R: Towards a science of cyber-physical systems design. In: Proc. 2nd ACM/IEEE Int'I Conf. Cyber-Physical Systems, pp. 99-108. IEEE Comput. Soc., Los Alamitos (2011)

6. Marculescu, R, Bogdan, P: Cyberphysical systems: workload modeling and design optimization. IEEE Des. Test Comput. 28(4), 78-87, July-August (2011)

7. Bogdan, P: Implantable pacemakers control and optimization via fractional calculus approaches: a cyber-physical systems perspective. In: Proceedings of the IEEE/ACM Third International Conference on Cyber-Physical Systems (ICCPS'12) (2012)

8. Balachandran, K, Sakthivel, R: Controllability of integrodifferential systems in Banach spaces. Appl. Math. Comput. 118, 63-71 (2001)

9. Balachandran, K, Sakthivel, R: Controllability of functional semilinear integrodifferential systems in Banach spaces. J. Math. Anal. Appl. 225, 447-457 (2001)

10. Fu, X: Controllability of abstract neutral functional differential systems with unbounded delay. Appl. Math. Comput. $151,299-314(2004)$

11. Ahmed, HM: Boundary controllability of nonlinear fractional integrodifferential systems. Adv. Differ. Equ. 2010, Article ID 279493 (2010)

12. Klamka, J: Stochastic controllability of linear systems with delay in control. Bull. Pol. Acad. Sci., Tech. Sci. 55(1), 23-29 (2007)

13. Klamka, J: Stochastic controllability of linear systems with state delays. Int. J. Appl. Math. Comput. Sci. 17, 5-13 (2007)

14. Klamka, J: Stochastic controllability of systems with variable delay in control. Bull. Pol. Acad. Sci., Tech. Sci. 56, 279-284 (2008)

15. Klamka, J: Stochastic controllability and minimum energy control of systems with multiple delays in control. Appl. Math. Comput. 206(2), 704-715 (2008)

16. Klamka, J: Stochastic controllability of systems with multiple delays in control. Int. J. Appl. Math. Comput. Sci. 19(1), 39-47 (2009)

17. Ahmed, HM: Controllability of fractional stochastic delay equations. Lobachevskii J. Math. 30, 195-202 (2009)

18. Sakthivel, R, Ganesh, R, Suganya, S: Approximate controllability of fractional neutral stochastic system with infinite delay. Rep. Math. Phys. 70, 291-311 (2012)

19. Sakthivel, R, Ganesh, R, Ren, Y, Anthoni, SM: Approximate controllability of nonlinear fractional dynamical systems. Commun. Nonlinear Sci. Numer. Simul. 18, 3498-3508 (2013)

20. Sakthivel, R, Ren, Y: Approximate controllability of fractional differential equations with state-dependent delay. Results Math. 63, 949-963 (2013)

21. Ren, Y, Bi, Q, Sakthivel, R: Stochastic functional differential equations with infinite delay driven by G-Brownian motion. Math. Methods Appl. Sci. 36, 1746-1759 (2013)

22. Ren, Y, Dai, H, Sakthivel, R: Approximate controllability of stochastic differential system driven by a Levy process. Int. J. Control 86, 158-1164 (2013)

23. Sakthivel, R, Suganya, S, Anthoni, SM: Approximate controllability of fractional stochastic evolution equations. Comput. Math. Appl. 63, 660-668 (2012)

24. Sakthivel, R: Complete controllability of stochastic evolution equations with jumps. Rep. Math. Phys. 68, 163-174 (2011)

25. Mandelbrot, BB, Van Ness, JW: Fractional Brownian motions, fractional noises and applications. SIAM Rev. 10, 422-437 (1968)

26. Boufoussi, B, Hajji, S: Neutral stochastic functional differential equations driven by a fractional Brownian motion in a Hilbert space. Stat. Probab. Lett. 82, 1549-1558 (2012)

27. Pazy, A: Semigroups of Linear Operators and Applications to Partial Differential Equations. Applied Mathematical Sciences, vol. 44. Springer, New York (1983)

28. Sakthivel, R: Approximate controllability of impulsive stochastic evolution equations. Funkc. Ekvacioj 52, 381-393 (2009)

10.1186/1687-1847-2014-113

Cite this article as: Ahmed: Approximate controllability of impulsive neutral stochastic differential equations with

fractional Brownian motion in a Hilbert space. Advances in Difference Equations 2014, 2014:113 